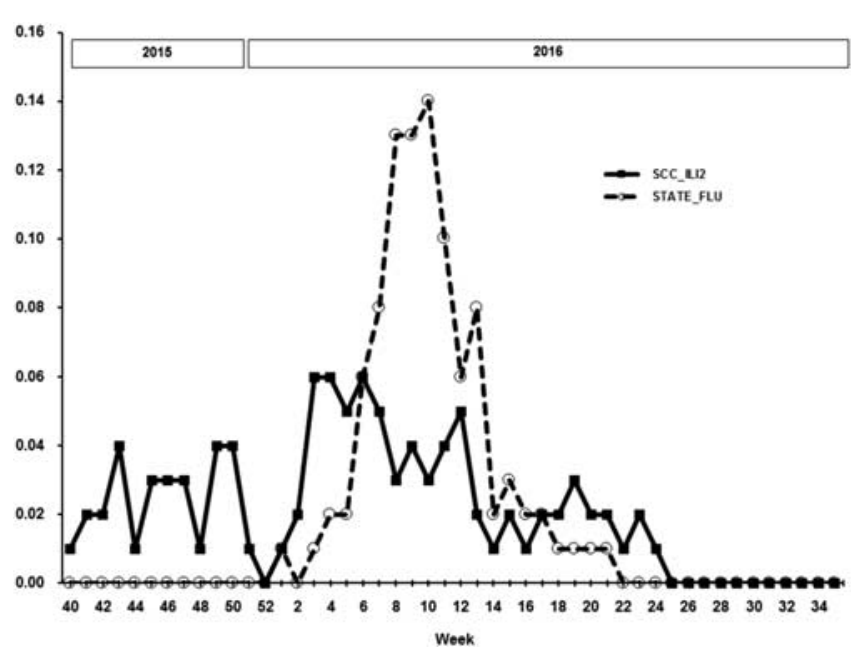

Figure 6. Epidemic Curves for Proportion of Flu Cases for State of Michigan and Sick Child Care: Year 3, 2015-2016.

Prehosp Disaster Med 2017;32(Suppl. 1):s192-s194

doi:10.1017/S1049023X17005076

Vulnerable Population Study of Household Injuries: A Case Study in Hong Kong

Nan Zhang ${ }^{1}$, Emily Y.y. Chan ${ }^{1}$, Chunlan Guo ${ }^{1}$, Janice Y. Ho ${ }^{2}$

1. Collaborating Centre For Oxford University And Cuhk For Disaster And Medical Humanitarian Response, The Chinese University of Hong Kong, Hong Kong/Hong Kong Prc

2. School Of Public Health And Primary Care, The Chinese University of Hong Kong, Hong Kong/Hong Kong Prc

Study/Objective: This study is primarily aimed to investigate the relationship among different types of household injuries with socio-demographic attributes in Hong Kong (an urban Chinese setting).

Background: Injury is a major global disease burden for the $21^{\text {st }}$ century. However, there is little research about unintentional household injury, especially in Asian urban areas.

Methods: A cross-sectional retrospective recall study was conducted in 2009 using a random telephone survey with a modified Chinese version of the World Health Organization Injury and Violence instrument. Ethics approval and participant's verbal consent were sought. The study samples included 6,570 non-institutionalized Cantonese-speaking Hong Kong residents of all ages and genders. Descriptive analysis and incidence rates were calculated for seven specific injuries, including dislocation and sprain, fracture, external injury, bruise, poisoning, burn and scald, and animal bites.

Results: In the previous 12 months upon the time of survey, 2,577 out of 6,570 respondents experienced household injuries. Among the seven types of injuries, bruise was reported with the highest incidence rate (25.3\%). Moreover, the probability of household injuries generally decreases with the increasing age from 40 years old. Gender was also confirmed to have influence in the household injuries. Females have a $17.1 \%$ higher rate than males when household injuries occur. There were district disparities of the household injury occurrence pattern.
Conclusion: Age, gender, and geographical location had strong relation with the incidence rate of household injuries. Further studies with a prospective longitudinal design should include injuries that happen outside of a household setting.

Prehosp Disaster Med 2017;32(Suppl. 1):s194

doi:10.1017/S1049023X17005088

The Disaster Risk Landscape for Small Island Developing States (SIDS)

James M. Shultz

Deep Center, University of Miami Miller School of Medicine, Miami/ FL/United States of America

Study/Objective: To examine disaster vulnerability of Small Island Developing States through a public health/socioecological lens.

Background: In contrast to continental nations, the world's 52 Small Island Developing States (SIDS) form a collective of countries that experience disproportionate challenges for sustainable development related their geography, small size, and physical isolation. SIDS also face elevated risks for disaster incidence and consequences, particularly in the realms of climate change, sea level rise, natural disasters (tropical cyclones, earthquakes, tsunamis, volcanoes), and marine hazardous materials spills. Cyclone Winston's impact on Fiji in 2016 and Cyclone Pam's landfall over Vanuatu in 2015 illustrate the special vulnerabilities of the SIDS.

Methods: The novel Disaster Risk Reduction (DRR) and Disaster Risk Management (DRM); challenges faced by SIDS were reviewed in light of United Nations guidance, the Sendai Framework, and the Sustainable Development Goals.

Results: For SIDS, the disaster risk landscape is shaped by several unique features: 1) small size and correspondingly limited resources; 2) elevated disaster frequency and severity based on geography (tropical latitude/longitude), geophysics (seismicity, volcanic activity, proximity to tectonic plate boundaries), and topography (sea level elevation, $360^{\circ}$ coastal perimeter, steep terrain on some islands); and 3) physical isolation from other nations - precisely because SIDS are individual islands or clusters of islands. For SIDS, the trifecta of natural disaster vulnerability, climate change, and rising ocean levels act synergistically to exacerbate disaster risks.

Conclusion: Dispersed broadly throughout the oceans of the world, the SIDS act inadvertently as an early warning network for detecting the initial signs of insidious global threats. Given these realities, DRR and DRM strategies must be tailored to the unique constellation of disaster hazards, and vulnerabilities that characterize the SIDS. The ability of SIDS to form robust alliances among counterpart island nations, is an urgent imperative as is the need for infusion of international support to enhance disaster resilience.

Prehosp Disaster Med 2017;32(Suppl. 1):s194

doi:10.1017/10.1017/S1049023X1700509X

\section{Reducing Non-communicable Disease Exacerbation after} a Disaster

Benjamin J. Ryan ${ }^{1}$, Richard C. Franklin ${ }^{1}$, Frederick M. Burkle, Erin C. Smith ${ }^{3}$, Peter Aitken ${ }^{1}$, Kerrianne Watt ${ }^{1}$, Peter A. Leggat ${ }^{1}$ 